\title{
Phronesis
}

\section{Le praticien réflexif}

Mythe ou réalité en formation à l'enseignement?

\section{Enrique Correa Molina et Lynn Thomas}

Volume 2, numéro 1, janvier 2013

Le praticien réflexif : mythe ou réalité en formation à

l'enseignement?

URI : https://id.erudit.org/iderudit/1015634ar

DOI : https://doi.org/10.7202/1015634ar

Aller au sommaire du numéro

Éditeur(s)

Institut de recherche sur les pratiques éducatives

ISSN

1925-4873 (numérique)

Découvrir la revue

Citer ce document

Correa Molina, E. \& Thomas, L. (2013). Le praticien réflexif : mythe ou réalité en formation à l'enseignement ? Phronesis, 2(1), 1-7.

https://doi.org/10.7202/1015634ar d'utilisation que vous pouvez consulter en ligne.

https://apropos.erudit.org/fr/usagers/politique-dutilisation/ 


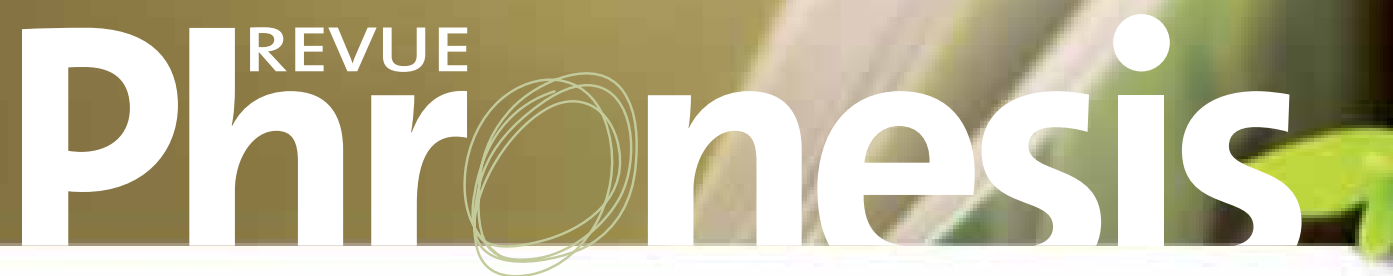

Institut de recherche sur les pratiques éducatives de l'Université de Sherbrooke

\section{LE PRATICIEN RÉFLEXIF :}

Enrique CORREA MOLINA et Lynn THOMAS

Université de Sherbrooke

2500 boul. de l'Univerisité

Sherbrooke (Québec)

Canada, J1K 2R1

Enrique.Correa.Molina@usherbrooke.ca

Lynn.Thomas@usherbrooke.ca 
Institut de recherche sur les pratiques éducatives de l'Université de Sherbrooke

Depuis les travaux de Schön (1983), le paradigme du praticien réflexif occupe une place importante dans la recherche en sciences de léducation et dans la formation professionnelle des enseignants. Depuis donc trente ans, la réflexion est considérée sur la scène internationale comme une caractéristique du «bon » professionnel. C’est donc une conséquence logique que dans le courant de professionnalisation de l'enseignement des initiatives aient été prises pour l'insérer dans les dispositifs de formation initiale et continue des enseignants. Nonobstant, cette intention de former le futur enseignant ou la future enseignante à la réflexion, visée louable en soi, nous pouvons nous interroger sur la signification de certains concepts, comme ceux de réflexion, de réflexivité, d'analyse réflexive ou de praticien réflexif, convoqués dans le contexte de la formation initiale sous un angle normatif comme, par exemple, le référentiel de formation à l'enseignement du Québec. Ce questionnement s’appuie d’abord sur le caractère polysémique entourant le concept de réflexion qui a été déjà relevé par divers travaux (Beauchamp, 2006 ; Correa Molina, Collin, Chaubet \& Gervais, 2010 ; Desjardins, 2000; Saussez \& Allal, 2007 ; Tardif, Borges \& Malo, 2012). Il s’appuie ensuite dans la diversité de dispositifs censés la développer, tels que l'utilisation de multimédia (Welsch \& Devlin, 2007), du mentorat (Sundli, 2007), de la recherche-action (Moran, 2007), du portfolio (Groom \& Maunonen-Eskelinen, 2006), etc., et dans la diversité de façons de l'envisager soit sous l'angle des perspectives (Grimmett, Erickson, Mackinnon, \& Riecken, 1990), des types (Valli, 1997), des dimensions et catégories (Louden, 1991), des niveaux (Van Manen, 1977 ; Sparks-Langer, Simmons, Pash, Colton \& Starko, 1990), des logiques (Beauchamp, 2006).

Que la réflexion soit une démarche jouant un rôle important dans le développement professionnel n’est pas a priori discutable. En effet, des travaux de recherche ont montré comment la réflexion pouvait être considérée comme une compétence transversale servant de levier au développement des compétences liées à l’acte d’enseigner de stagiaires en enseignement (Gervais, Correa Molina \& Lepage, 2008). D’ailleurs, dans un ouvrage tout récent, Tardif, Borges \& Malo (2012) relèvent que, dans le monde intellectuel, les travaux de Schön (1983) sur le praticien réflexif ont provoqué au moins trois grandes percées : une première de nature critique, car ces travaux remettent en question la vision instrumentale de la formation professionnelle à lépoque; une deuxième de nature heuristique-méthodologique, car les études de cas utilisés par Schön montrent le caractère idiosyncrasique de l'activité professionnelle; finalement, une troisième percée de nature scientifique, car celle-ci met en relief l'intelligibilité professionnelle et donc sa dimension cognitive. Nous insistons donc en ce que la réflexion et le paradigme du praticien réflexif n'est pas questionné ici. Ce qui pose problème semble davantage être l'utilisation et l'usage quasi systématique de ce concept en contexte de formation initiale. Cest aussi le fait que les programmes de formation initiale et continue peuvent proposer de mettre à disposition des outils, ou des dispositifs annoncés comme porteurs de démarches réflexives susceptibles de développer la réflexion chez les futurs professionnels sans que nécessairement ceux-ci répondent aux objectifs de contribuer à former un "praticien réflexif et critique ». En vérité, ces outils, ces démarches ou dispositifs ne semblent pas toujours être en mesure de dépasser le niveau de description ou de justification d'une pratique éducative (Correa Molina et Gervais, 2010). En ce sens, par létude quelle a réalisée, Beauchamp (2006) nous signale que le raisonnement qui sous-tend la réflexion peut être de divers ordres. Selon elle, réfléchir peut signifier : penser différemment ou plus clairement; justifier ses choix; examiner ses actions ou décisions; changer sa pensée ou sa connaissance; améliorer l’action; améliorer lapprentissage des élèves; se transformer en tant qu'individu et, ainsi, transformer la société.

Rappelons qu’à l'époque où il émerge, le paradigme du praticien réflexif a trouvé un terreau fertile pour prospérer en formation à l’enseignement, car le mouvement de professionnalisation était déjà en marche (Tardif, Borges \& Malo, 2012). Nombreux sont en effet le chercheur à s'y intéresser, dont Zeichner, Calderhead, Van Manen ou Fenstermacher, pour ne nommer que quelques icônes de la littérature à ce sujet. Cette effervescence pour ce paradigme a touché la formation professionnelle à lenseignement à un point tel que l’on a vu émerger des référentiels de compétences, prescrits par des gouvernements, où la dimension réflexive en fait partie. À titre d’exemple, mentionnons le référentiel de compétences orientant et soutenant la formation à l’enseignement au Québec (Ministère de l'Éducation du Québec, 2001) 
qui stipule explicitement quau terme de la formation initiale létudiant doit être en mesure de mener une « démarche d'analyse réflexive de manière rigoureuse sur des aspects précis de leur enseignement » (p. 129). Pourtant, cette même prescription ne signale guère sous quelle conception de la réflexion se situe-t-on, ni de quelle manière peut-on lopérationnaliser ni lévaluer objectivement puisque, à la fin de la formation, les programmes doivent en garantir son développement.

Nous ne renions pas l'importance de la réflexion en contexte de formation. Bien au contraire, nous prônons pour une formation qui stimule la réflexion des futurs enseignants. Il faut cependant considérer qu'inciter à la réflexion et au développement d'une pratique réflexive, en contexte de formation initiale à l'enseignement, est une démarche complexe, car celle-ci suppose la mobilisation de valeurs, d'attitudes, de croyances et le développement d'habiletés cognitives qui évolueraient sur une longue période de temps (Calderhead \& Gates, 1993 ; Korthagen \& Vasalos, 2005 ; Pultorak, 1996). Elle semble donc s'inscrire dans un long processus d'insertion et de développement professionnel.

Compte tenu de ce qui précède, vouloir développer la réflexion en contexte de formation professionnelle conduit nécessairement à la prise en compte, tant de la complexité que de la durée d'un tel processus. Les curriculums de formation à l'enseignement doivent donc non seulement offrir des activités présumées soutenir le développement de telle compétence, mais aussi de le vérifier. Mais pouvons-nous être certains que c'est à partir de ces activités que les futurs enseignants ont réussi à construire et à développer leur réflexivité? Ces activités bénéficient-elles équitablement à tous ou davantage à ceux et celles qui ont une plus grande habileté verbale et/ou descriptive ou qui sont davantage en mesure d'expliciter leurs pratiques? Ces questions ne manquent pas de pertinence, car sans que l'on puisse mettre en doute l'impact du paradigme du praticien réflexif en formation professionnelle, on ne peut pas dire autant de sa compréhension (Tardif, Borges \& Malo, 2012).

En tant que formateurs et chercheurs participant au processus formatif des futurs enseignants, nous sommes donc interpellés par ces questions qui touchent le paradigme du "praticien réflexif ». Ce numéro thématique, découlant de deux journées détude tenues en 2011, a la particularité de focaliser l'aspect de la réflexivité en formation initiale à l'enseignement sous des angles différents. Il réunit des textes traitant de cette dimension qui traverse la formation professionnelle des futurs enseignants, en considérant des travaux de chercheurs venant d'horizons divers du Canada tels que l'Alberta, la Colombie-Britannique, le Manitoba, le Québec et l'Ontario.

Comme nous le disions précédemment, que la réflexion joue un rôle dans le développement professionnel des enseignants lors de leur formation initiale n'est pas remis en question. Néanmoins, ce concept éveille la critique et invite au dialogue pour en saisir sa signification. Nous vous invitons à lire les divers textes qui constituent ce numéro et qui traitent du sujet sous des angles fort divers, voire éclectiques, ce qui, à notre avis, constitue sa richesse et son intérêt. Deux grandes sections organisent les différents textes de ce numéro thématique. La première section que nous avons appelée Regards croisés sur le concept réunit des textes d’ordre théorique et argumentatif. Que ce soit à travers une critique du concept, un questionnement sur les bienfaits ou dommages du paradigme du praticien réflexif, une dialectique historique descriptive entre chercheurs sur lévolution de leur compréhension du concept ou à travers une perspective sociale ou de développement professionnel, les auteurs nous parlent ici de leurs postures, leurs visions, leurs arguments et même leurs recommandations pour faire de la réflexion une partie prenante de la formation initiale et un moteur de développement professionnel.

La deuxième partie réunit des textes d’ordre empirique qui, entre autres, nous parlent des dispositifs supposés contribuer au développement de la réflexion, des difficultés méthodologiques pour appréhender un concept polysémique tel que celui de réflexion en contexte de formation initiale à l'enseignement.

\section{Regards croisés sur le concept}

Mettre à distance la sympathie que ce concept évoque en chacun pour en saisir sa juste mesure. Voilà à quoi nous appelle le texte de Couturier (dans ce numéro). C'est sous le regard du sociologue que cet auteur nous expose ses idées encadrées par son titre critique de la réflexion (estce donc possible?). Est-il possible de critiquer la réflexivité? Est-ce donc possible de faire preuve de réflexivité? Quoi qu'il en soit, l'auteur s'appuie sur de solides références pour soulever divers aspects de la réflexivité, dont sa dimension collective et, ce qui n’est pas moindre, la condition sine qua non, pour faire d'elle un objet de formation professionnelle, à savoir le refus de la réduire au retour de soi sur lui-même. À la fin de son analyse, Couturier nous convie à concevoir la réflexivité à travers cinq plans logiques non pas isolés, mais irréductiblement entrelacés les uns aux autres, dont le dernier touche directement les métiers ou professions où le rapport à l'autre est de mise : le plan de la 
médiation sociale.

De manière provocatrice, Russell (dans ce numéro) se demande si la pratique réflexive n’aurait-elle causé plus de dommage que de bien dans la formation des enseignants. D'ailleurs, il commence son texte en se demandant pourquoi sempresse-t-on si tant avec la pratique réflexive en formation initiale à l’enseignement si, semble-t-il, il n’y a pas d’évidence convaincante qui montre que la réflexion améliore la qualité des enseignants. Prenant appui dans sa vaste expérience en formation initiale à l'enseignement, Russell soulève sa préoccupation quant au manque de guide ou d'orientation donnée aux étudiants sur ce qui signifie de réfléchir et sur la tendance de séparer la réflexion du contexte de laction de la personne. L'auteur remarque aussi le peu ou pas de connexion entre les cours universitaires et les expériences de terrain lors des stages des futurs enseignants, et le peu d’écrits faisant le lien entre stages et réflexion. C’est le slogan du " praticien réflexif » qui, selon Russell, occasionne plus de dommage que du bien dans la formation initiale, car, à force de demander de réfléchir ici et là dans les différentes instances du programme de formation, les étudiants finissent pour se lasser. De plus, quand, entre autres, les formateurs ne prennent pas le temps d'expliquer ce qui signifie pour eux la réflexion, qu'ils ne sont pas des modèles de réflexion et qu'ils n'arrivent pas à lier de manière claire réflexion et développement professionnel, il n’est pas surprenant que l'appel à être un "praticien réflexif » demeure quelque chose d'abstrait. Russell affirme que c'est important d'aider les futurs enseignants à voir la pratique réflexive comme un processus pouvant lier l'art de l'enseignement avec les sciences de léducation. C’est tout un défi pour la formation initiale. Mais si nous voulons que les futurs enseignants s'habituent à connecter leur pratique avec la théorie, c'est un défi que nous nous devons de relever.

Pour leur part, d'une manière originale, mais non pas moins rigoureuse, MacKinnon, Clarke et Gaalen (dans ce numéro) nous partagent lévolution de leur compréhension du concept de réflexion à travers leurs 25 à 30 années de carrière. Délibérément ils utilisent une approche conversationnel et chronologique pour exposer leurs idées en soulignant tant leur interdépendance que ses origines épistémiques. En commençant avec leur rencontre avec Schön dans les années 80, lors de la visite de ce dernier à l'Université de la Colombie-Britannique, en 1986, avant que son nom soit bien connu par les formateurs et chercheurs en formation à l'enseignement, ces auteurs discutent de l'influence de Schön sur la recherche en enseignement en et sur leur propre pratique en tant que formateurs. Ils touchent divers points, tels que la formation des enseignants, l'autoanalyse, la réflexion comme dynamique individuelle et sociale, les défis d'une épistémologie de la pratique, entre autres. Ils concluent leur dialogue en soulevant quelques aspects à améliorer dans les programmes de formation comme, par exemple, le manque de cohérence entre cours académiques et expériences de terrain, car, dans ce scénario, on laisse aux étudiants la responsabilité de la conceptualisation de leurs apprentissages en terrain. Ils encouragent donc un engagement actif entre université et formateurs du milieu pour surmonter ce manque de cohérence. Ils s’accordent pour signaler que, malgré le fait que Schön ne faisait pas de recherche ni en éducation ni en formation à l'enseignement, ses écrits sur la pratique réflexive et l'épistémologie de la pratique ont eu plus d'influence sur ce domaine que n’importe quel autre chercheur.

En prenant appui sur une recension d'écrits prenant en compte des études et des exemples de pratique, Lison, dans ce numéro, argumente en faveur d'une formation à la réflexivité visant les futurs enseignants et pensée sous l'angle du développement professionnel. En se faisant lécho du caractère polysémique de la réflexivité, partagé par divers auteurs de ce numéro, Lison défend le modèle de Kelchterman (2001) qui définit la pratique réflexive selon quatre dimensions : instrumental, morale, politique et affective. En soulignant l'importance de l'accompagnement pour dépasser les limites d'une réflexion in solo, l'auteure voit la pratique réflexive comme un moyen susceptible de réduire lécart entre ce que les jeunes enseignants construisent dans et par les cours à l'université et les situations expérienciées qu'ils vivent dans les stages.

Falkenberg (dans ce numéro) postule que les programmes de formation à l'enseignement ont le mandat d'encourager la responsabilité sociale des futurs enseignants et qu'une des façons d'y parvenir, c'est en les aidant à développer des qualités personnelles comme la compassion et l'empathie au moyen de la réflexion. Dans son texte, cet auteur s'interroge sur quel type de pratique réflexive ferait du praticien un meilleur enseignant par rapport à la responsabilité sociale qui lui revient. Falkenberg, professeur à l'University of Manitoba, donne l'exemple de cas récents d'intimidation pour mettre en lumière le besoin de mieux former les enseignants à réagir avec empathie à ce type de situations. Le mandat de responsabilité sociale de léducation est, selon lui, un mandat d'amélioration de la société en s'attaquant à l'iniquité sociale, le manque de tolérance, d’engagement démocratique, etc. Falkenberg argumente que la pensée réflexive et la pratique réflexive jouent un rôle important en orientant le mandat de responsabilité social de la formation à l'enseignement à travers le développement des qualités personnelles de l'individu, car cela implique des changements dans l'identité professionnelle et le comportement de celui-ci. En ce sens, lauteur suggère quelques démarches en fonction de quatre dimensions de la pratique réflexive : létendue, la profondeur, la temporalité 
interne et la temporalité externe. Falkenberg conclut en disant que les programmes de formation à lenseignement ont un mandat de former des enseignants avec un bon sens de leur responsabilité morale envers leurs élèves et la société en général et, d’après lui, la réflexivité est une avenue prometteuse pour y parvenir.

\section{Des données empiriques}

Trois textes s’appliquent ici à déployer de manière empirique la façon dont la réflexion est travaillée dans le contexte de la formation initiale des enseignants. Quoique menées de façon différente, ces trois études touchent d'une manière ou d'une autre le support que l'on met à disposition de futurs enseignants pour réfléchir. Que ce soit au moyen de dispositifs, tels que le portfolio (Nickel, dans ce numéro) et l'interaction en ligne (Collin, dans ce numéro), ou de l'accompagnement d'un tuteur (Chaubet, Correa Molina \& Gervais, dans ce numéro), ces auteurs présentent des résultats de nature qualitative quant à l’apport de ces dispositifs, à la variation de la réflexion dépendant du contexte académique ou pratique où elle se réalise, à la façon dont les tuteurs repèrent si une personne stagiaire réfléchit ou pas.

Parmi léventail de dispositifs utilisés pour encourager la réflexion (Welsch \& Devlin, 2007; Sundli, 2007; Moran, 2007; Groom \& MaunonenEskelinen, 2006), Nickel (dans ce numéro) se penche sur le portfolio comme un outil encourageant le regard critique que l'on prétend développer chez les futurs enseignants. Participant à la formation des enseignants à l'Université Mont-Royal, à Calgary, elle décrit son étude sur le développement de la capacité à réfléchir chez les étudiants en formation à l’enseignement quand ils autoévaluent leurs propres portfolios de réflexion. Elle note que malgré le fait que l’objectif des portfolios était d’aider les enseignants en formation à évaluer le développement de leurs compétences en enseignement, plusieurs ont aussi fait un lien entre les artefacts qu'ils choisissaient et leurs profils professionnels. Nickel résume les apprentissages de ses étudiants dans des catégories liées à la planification, la promotion de l’apprentissage chez les élèves, lévaluation, l'environnement de la classe, et le développement d'une identité professionnelle chez les futurs enseignants. Comme ce dernier aspect s'est révélé une retombée importante des portfolios, Nickel a décidé de raffiner l'objectif des portfolios afin de mettre l'accent sur lémergence de leur identité professionnelle et, conséquemment, de mieux cadrer la structure de cet outil.

Collin (dans ce numéro) se penche sur un dispositif académique utilisé auprès de stagiaires finissants du programme de formation à l’enseignement secondaire pour soutenir leur pratique réflexive. En interviewant des stagiaires et des superviseurs, lauteur cherche à mieux comprendre la variation de la pratique réflexive des stagiaires selon les contextes où ils déploient leur action, à savoir le milieu universitaire ou académique et le milieu scolaire ou pratique. Ces variations sont mises en évidence en termes de motivation et finalité, en termes d’acteurs du stage d'enseignement et en termes de modalités d'interaction. Les résultats obtenus par létude de Collin permettent d'envisager une double conception de la pratique réflexive chez les stagiaires où celle réalisée en contexte pratique aurait comme finalité l'amélioration de leur enseignement et celle réalisée en contexte académique serait plutôt orientée par lévaluation à laquelle ils doivent se soumettre. Les variations observées en termes d'acteurs et en termes d'interaction, soulèvent la question du comment manier ces deux types de réflexion en contexte de formation initiale. Plutôt que de voir ces variations comme un obstacle, Collin les voit davantage comme étant complémentaires.

Létude menée par Chaubet, Correa Molina et Gervais (dans ce numéro), s’attarde à la façon dont on aborde la compétence à réfléchir ou à faire réfléchir en contexte de formation initiale à l’enseignement. Dans un premier temps, basés sur une étude à caractère longitudinale (Correa Molina \& Gervais, 2012) sur le développement de la réflexivité chez de futurs enseignants, les auteurs s'interrogent sur les repères utilisés par les tuteurs de stagiaires pour dire si leur stagiaire réfléchit ou pas. Létude ne manque pas d'intérêt, car, d'une certaine manière, elle met en lumière la question de lévaluation de la réflexion selon des grilles personnelles des tuteurs qui accompagnent, suscitent et évaluent la réflexion du stagiaire. Dans un deuxième temps, ils testent la validité méthodologique desdits repères pour, finalement, tester la robustesse des grilles de catégories des tuteurs. Tout en soulevant les limites de leur étude, Chaubet, Correa Molina et Gervais concluent que la catégorisation pragmatique des tuteurs, définie par eux en termes de "capacités ", ne serait que les pointes de l'iceberg. Ce qui, évidemment, ne reflète pas ni la nature ni l’ampleur des processus que la recherche future pourrait élucider.

En guise de conclusion 
Afin d'introduire les différents textes qui composent ce numéro thématique, nous avons titré l'introduction de celui-ci « Le praticien réflexif : mythe ou réalité en formation à l'enseignement? » N’est qu’après avoir présenté chacun des textes qui composent ce numéro que ce titre (et question à la fois) trouve sons sens. Mythe ou réalité? Voilà la question! Tardif, Borges et Malo (2012) ont dirigé un ouvrage qui essaie de faire le point sur la réflexion 30 ans après le boom Schönien. Tant les auteurs qui participent de cet ouvrage comme ceux qui composent celuici coïncident sur le fait que le paradigme du "praticien réflexif » est loin de faire unanimité, car il est basé sur le concept de la réflexion qui se trouve à être polysémique et donc interprété de maintes façons. Sous cet angle et vu le caractère prescriptif de cadres référentiels en formation professionnelle qui voient le praticien réflexif comme un but du parcours formatif, nous pouvons être tentés de le considérer tout simplement comme un mythe. Nonobstant, si d'une part l'on prend en compte des études sur des dimensions de la formation initiale qui informent de certains résultats montrant que la réflexion existe bel et bien et qu’elle se développe dans ce contexte (Chaubet, Correa Molina et Gervais; Collin; Nickel) et que, d’autre part, les arguments, analyses critiques, recommandations et conditions pour encourager le praticien à réfléchir sur sa pratique et assumer sa responsabilité sociale (Couture; Falkenberg; MacKinnon, Clarke et Erickson; Lison; Russell) présentés par les auteurs de ce numéro, nous devons nous incliner au fait que réfléchir en contexte de formation initiale est tout à fait possible à condition de travailler pour que les dispositifs mis en place incitent les jeunes professionnels à assumer un rôle actif de développement professionnel en réfléchissant sur ce qu'ils font et en argumentant leur pratique plutôt que de voir la réflexion comme un travail universitaire de plus parmi les autres à rendre dans son parcours formatif.

Pour finir, disons que, d'une certaine manière, ce numéro reflète bien la diversité qui entoure le paradigme du praticien réflexif, car, de manière éclectique, il réussit à réunir dans un même volume les travaux de chercheurs d'horizons différents, tant par la langue que les systèmes de formation. Nous pouvons dire que, dans le cadre des journées détude dont cet ouvrage découle et de par ce numéro même, nous faisons preuve que pour réfléchir ensemble il n’y pas de si grandes barrières et qu’en fin de compte nous partageons toutes les mêmes préoccupations quant à la qualité de la formation des professionnels de demain. C’est justement cet éclectisme qui rend la lecture intéressante.

\section{Références}

Beauchamp, C. (2006). Understanding Reflection in Teaching: A Framework for Analyzing the Literature. (Thèse de doctorat inédite). Montréal : McGill University.

Calderhead, J. \& Gates P. (1993). Conceptualizing Reflection in Teacher Development. London : The Falmer Press.

Correa Molina, E. \& Gervais, C. (2012). Contenus alimentant la réflexion de stagiaires en début de formation. Dans M. Tardif, C. Borges \& A. Malo (dir.) Le virage réflexif en éducation. Où en sommes-nous 30 ans après Schön? (p. 195-213 ). Bruxelles : DeBoeck.

Correa Molina, E. \& Gervais, C. (2010). Developping Reflexive Competencies in Student Teaching: Which Theoretical Basis? XIV World Congress of Comparative Education Societies, 14-18 Juin 2010, Istanbul.

Correa Molina, E., Collin, S., Chaubet, P. \& Gervais, C. (2010). Concept de réflexion : un regard critique. Éducation et Francophonie. 38(2), 135-154.

Desjardins, J. (2000). Analyse critique du champ conceptuel de la formation réflexive. (Thèse de doctorat), Université de Montréal.

Gervais, C., Correa Molina, E. \& Leapge, M. (2008). Comment se construisent les compétences liées à l'acte d'enseigner? Explicitations de pratiques pendant les stages. Dans E. Correa Molina \& C. Gervais (dir.), Les stages en formation à l'enseignement. Pratiques et perspectives théoriques (p. 153-175). Québec : Presses de l'Université du Québec.

Grimmett, P., Erickson, G., Mackinnon, G. \& Riecken, T. (1990). Reflective practice in teacher education. Dans R. Clift, R. Houston et M. Pugach (dir.), Encouraging Reflective Practice in Education: An Analysis of Issues and Programs (p. 20-38). New York : Teachers College Press.

Groom, B. \& Maunonen-Eskelinen, I. (2006). The use of portfolios to develop reflective practice in teacher training: A comparative and collaborative approach between two teacher training providers in the UK and Finland. Teaching in higher education, 11(3), 291-300.

Korthagen, F. \& Vasalos, A. (2005). Levels in reflection : Core reflection as a means to enhance professional growth. Teachers and teaching: Theory and practice, 11(1), 47-71.

Louden, W. (1991). Understanding Teaching: Continuity and change in teachers' knowledge. New York : Teachers College Press Columbia University.

Ministère de l'Éducation (2001). La formation à l'enseignement : les orientations, les compétences professionnelles. Québec: Gouvernement du 
Québec.

Moran, M. J. (2007). Collaborative action research and project work: Promising practices for developing collaborative inquiry among early childhood preservice teachers. Teaching and teacher education: an international journal of research and studies, 23(4), 418-431.

Pultorak, E. (1996). Following the Developmental Process of Reflection in Novice Teachers: Three Years of Investigation. Journal of Teacher Education, 47 (4).

Saussez, F. \& Allal, L. (2007). Réfléchir sur sa pratique : le rôle de l'autoévaluation? Mesure et évaluation en éducation, 30(1), 97-124.

Schön, D.A. (1983). The reflective Practitioner. New York : Basic Book.

Sparks-Langer, G., Simmons, J., Pasch, M., Colton, A., \& Starko, A. (1990). Reflective pedagogical thinking: how can we promote it and measure it? Journal of Teacher Education, 41(5), 23-32.

Sundli, L. (2007). Mentoring-A new mantra for education? Teaching and teacher education: An international journal of research and studies, 23(2), 201-214.

Tardif, M., Borges, C. \& Malo, A. (2012). Le virage réflexif en éducation : où en sommes-nous 30 ans après Schön? Bruxelles : DeBoeck.

Valli, L. (1997). Listening to other voices: a description of teacher refelection in the Unites States. Peabody Journal of Education. 72(1), 67-88.

Van Manen, M. (1977). Linking ways of knowing with ways of being practical. Curriculum Inquiry. 6, 205-228.

Welsch, R. \& Devlin, P. (2007). Developing preservice teachers'reflection: Examining the use of video. Action in teacher education, 28(4), 53-61. 